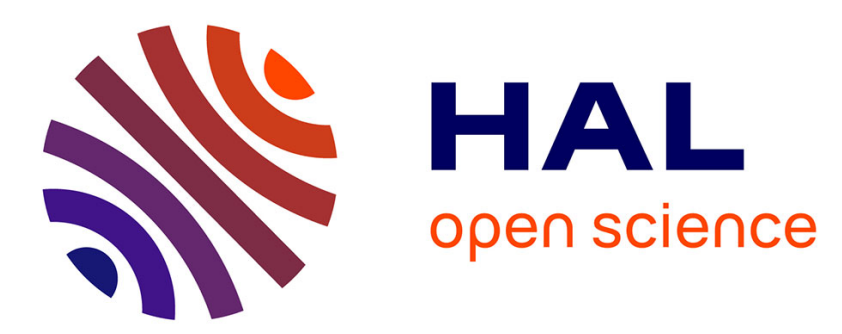

\title{
Les vestiges antiques de Rennaz-Noville (Vaud, Suisse) et leur contexte sédimentaire: nouvelles évidences de l'écroulement du Tauredunum
}

\author{
Morgan Millet, Michel Guélat
}

\section{- To cite this version:}

Morgan Millet, Michel Guélat. Les vestiges antiques de Rennaz-Noville (Vaud, Suisse) et leur contexte sédimentaire: nouvelles évidences de l'écroulement du Tauredunum. Archimède: archéologie et histoire ancienne, 2020, 7, pp.176-187. halshs-02893457

\section{HAL Id: halshs-02893457 \\ https://shs.hal.science/halshs-02893457}

Submitted on 8 Jul 2020

HAL is a multi-disciplinary open access archive for the deposit and dissemination of scientific research documents, whether they are published or not. The documents may come from teaching and research institutions in France or abroad, or from public or private research centers.
L'archive ouverte pluridisciplinaire HAL, est destinée au dépôt et à la diffusion de documents scientifiques de niveau recherche, publiés ou non, émanant des établissements d'enseignement et de recherche français ou étrangers, des laboratoires publics ou privés. 


\section{ARCHIMÈDE N N 7}

\section{DOSSIER THÉMATIQUE : GESTES RITUELS. DE LA TRACE À L'INTERPRÉTATION}

\section{ACTUALITÉ DE LA RECHERCHE GÉOSCIENCES ET ARCHÉOLOGIE : INTERACTIONS, COMPLÉMENTARITÉS ET PERSPECTIVES}

114 Bruno GAVAZZI

Heureuses rencontres. Vers le développement d'approches intégrées en géosciences et archéologie

119 Jean-Paul BRAVARD

Dialogue interdisciplinaire : de I'unité stratigraphique aux interactions culture-environnement

129 Ferréol SALOMON

Les origines d'Ostie : quelles interactions avec la dynamique d'embouchure? (Delta du Tibre, Italie)

141 Quentin BORDERIE, Rowena Y. BANERJEA, Stéphane BONNET, Yannick DEVOS, Cristiano NICOSIA, Christophe PETIT, Ferréol SALOMON, Nathalie SCHNEIDER, Barbora WOUTERS, \& Patrice WUSCHER Géoarchéologies des contextes urbains : mieux comprendre les modalités de l'artificialisation des géosystèmes

158 Patrice WUSCHER, Christophe JORDA, Quentin BORDERIE, Nathalie SCHNEIDER \& Laurent BRUXELLES De la formation géologique à la tranchée : trouver et comprendre les sites archéologiques menacés par les travaux d'aménagement du territoire

176 Morgan MILLET \& Michel GUÉLAT

Les vestiges antiques de Rennaz-Noville (Vaud, Suisse) et leur contexte sédimentaire : nouvelles évidences de l'écroulement du Tauredunum

188 Théophile PIAU, François BÉTARD, Fabienne DUGAST, Gilles ARNAUD-FASSETTA \& Vincent VIEL Dynamique géomorphologique holocène et occupation humaine dans le bassin-versant de l'Eure (Bassin de Paris, France) : potentiels d'une approche géoarchéologique multiscalaire et diachronique

205 Dominique SCHWARTZ, Vincent ROBIN, Pierre ADAM, Philippe SCHAEFFER, Anne GEBHARDT, Pierre-Alexis HERRAULT, Benjamin KELLER, Daniele DAPIAGGI, Claire STEVENEL, Maxime THISS, Martine TRAUTMANN \& Damien ERTLEN

Les géosciences au service de l'archéologie agraire. Une étude de cas sur les rideaux de culture de Goldbach (68)

217 Étienne MANTEL, Stéphane DUBOIS, Jonas PARÉTIAS, Victor VISQUESNEL-SCHLOSSER, Corentin VOISIN, Bruno GAVAZZI \& Matthieu RICHARD

Étudier I'occupation d'une ville : les enjeux du PCR « Topographie générale et insertion territoriale de l'agglomération antique de Briga »

231 Charlène MOREL

Un modèle pour comprendre l'influence de l'état de la recherche, des processus post-déposition et de l'attractivité sur la découverte des sites archéologiques dans le Kochersberg (Bas-Rhin, Alsace, Grand-Est, France)

241 Lizzie SCHOLTUS

Spatialisation des découvertes, modélisation du passé. L'informatique au service de l'archéologie

254 Guillaume HULIN \& François-Xavier SIMON

Inrap et géophysique : vers une approche raisonnée

260 Hugo REILLER, Matthieu FUCHS, \& Bruno GAVAZZI

Approche multi-méthodes expérimentale pour l'étude d'un site d'occupation romaine et médiévale à Horbourg-Wihr

272 François-Xavier SIMON, Julien GUILLEMOTEAU, Guillaume HULIN, Joachim RIMPOT, Julien THIESSON \& Alain TABBAGH

De nouvelles perspectives pour les applications des méthodes électromagnétiques basse fréquence en archéologie

283 Rémy WASSONG \& Bruno GAVAZZI

Apport des prospections magnétiques haute résolution à la compréhension d'un habitat protohistorique : l'exemple du site de hauteur fortifié du Maimont 


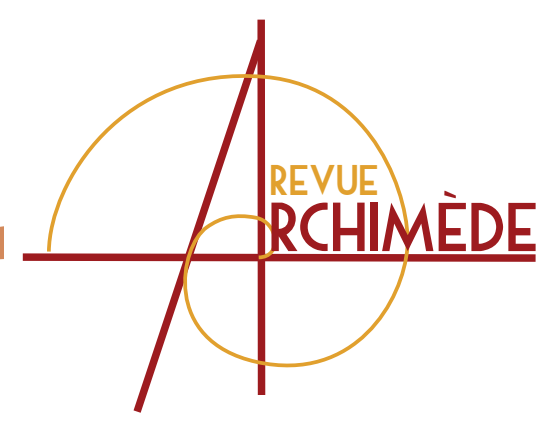

\title{
LES VESTIGES ANTIQUES DE RENNAZ-NOVILLE (VAUD, SUISSE) ET LEUR CONTEXTE SÉDIMENTAIRE : NOUVELLES ÉVIDENCES DE L'ÉCROULEMENT DU TAUREDUNUM
}

\author{
Morgan MILLET ${ }^{1}$, Michel GUÉLAT² \\ ${ }^{1}$ Archeodunum SA, m.millet@archeodunum.ch \\ ${ }^{2}$ Sediqua Géosciences sarl, michel.guelat@sediqua.ch
}

\section{RÉSUMÉ}

Des vestiges antiques ont été découverts à RennazNoville, dans le sud-ouest de la Suisse, au débouché de la vallée du Rhône dans le lac Léman. Les coupes dégagées lors de la construction d'un canal ont révélé que le sommet de l'accumulation sédimentaire se compose d'un diamicton déformé et compartimenté reposant, selon une surface de cisaillement, sur des dépôts limniques. Inclus à la masse transportée, des paléosols renfermant des artefacts ont subi une déformation d'amplitude variable. Une centaine de structures archéologiques ont été mises au jour dans l'un d'entre eux, auxquelles s'ajoutent des maçonneries en position secondaire. Le mobilier collecté et les datations ${ }^{14} \mathrm{C}$ convergent vers une occupation comprise entre le I $^{\text {er }}$ et la fin du IV siècle ap. J.-C. Une occupation du Second âge du Fer, oblitérée par l'occupation antique, est également concernée par les mouvements de terrain. Tous les éléments de datation obtenus sont compatibles avec un glissement déclenché vers le milieu du

\section{MotS-CLÉS}

Plaine alluviale,

Rhône,

glissement,

paléosols,

époque gallo-romaine,

La Tène,

maçonneries,

incinérations,

sources historiques.
$\mathrm{VI}^{\mathrm{e}}$ siècle par l'écroulement

dit du Tauredunum, en 563

ap. J.-C. Outre des éléments de chronologie inédits, les découvertes à Rennaz-Noville fournissent ainsi une nouvelle argumentation sur les effets de cette catastrophe en milieu terrestre.
Ancient remains have been discovered in RennazNoville, in southwestern Switzerland, where the Rhône Valley opens onto Lake Geneva. The stratigraphic sections documented during the construction of a canal revealed that the sedimentary sequence consists of a deformed and compartmentalized diamicton based, according to a thrust plane, on limnic deposits. Paleosols containing artifacts included in the transported mass have been deformed to varying degrees. About one hundred archaeological structures have been discovered in one of these with, in addition, masonry in a secondary position. The collected artifacts and ${ }^{14} \mathrm{C}$ dates converge towards an occupation between the $1^{\text {st }}$ and the end of the IVth century AD. Ground movements also affect a Late Iron Age occupation, largely obliterated by the gallo-roman settlement. These facts are compatible with a landslide triggered in the middle of the $\mathrm{VI}^{\text {th }}$ century, relating to the so-called Tauredunum event in 563 AD. In addition to unpublished chronological elements, the discoveries at Rennaz-Noville thus provide new evidence on the effects of the disaster on the terrestrial environment.

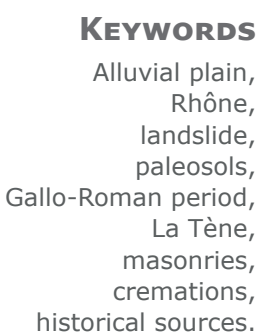

KeYwORDS Rhône, landslide, paleosols, La Tène, masonries, historical sources. 
La construction du canal du Haut-Lac, une infrastructure d'évacuation des eaux dans le Chablais vaudois (Suisse), a impliqué une intervention archéologique à l'est de Noville, près de Rennaz. Ce village se situe dans la plaine du Rhône, en rive droite, plus précisément à $1,7 \mathrm{~km}$ du lit actuel du fleuve et à 1,6 km au sud du lac Léman (fig. 1). Des sondages exploratoires sur l'emprise ont permis de reconnaître une section [1] recelant du mobilier et des structures archéologiques. D'une altitude moyenne de $376 \mathrm{~m}$, cette zone allongée dans I'axe nord-sud se trouve donc au milieu du fond de vallée, large de $6 \mathrm{~km}$. Elle mesure $200 \mathrm{~m}$ de long pour $20 \mathrm{~m}$ de large environ. À ce même endroit, le remplissage sédimentaire, jusqu'à la roche encaissante, atteint 790 m d'épaisseur environ [2] et se compose schématiquement de trois unités. Sous la surface se succèdent des formations fluviatiles qui recouvrent des sédiments lacustres, eux-mêmes sus-jacents à un till extrêmement compacté, en contact avec le fond rocheux [3]. Mais au voisinage du site et dans toute la partie centrale de la plaine apparaît en surface une unité supplémentaire, qui engendre les collines de Chessel-Noville [4]. Des travaux récents ont révélé que ces dépôts chaotiques de type diamicton [5] correspondent à la partie distale du matériel d'un écroulement [6]. Pour rappel, cette catastrophe se serait produite au sud-ouest de la plaine, sur le flanc de La Suche, un promontoire formé par les nappes préalpines. Elle pourrait bien coïncider avec l'écroulement dit du Tauredunum, qui a eu lieu en 563 ap. J.-C. selon des sources écrites et qui a déclenché un raz de marée dans le lac Léman [7]. Il s'agit de la Chronique de Marius, évêque d'Avenches, datée de 573 ap. J.-C., et de I'Histoire des Francs rédigée par Grégoire de Tours à partir de 583. Leurs auteurs y décrivent l'événement, survenu selon eux à la même date de 563 , mais via deux scénarios divergents. Pour Marius d'Avenches, il s'agit de l'effondrement d'un pan de la montagne éponyme qui surplombe le Rhône. S'abattant sur le bourg en contrebas, il le détruit, de même que l'ensemble des villages environnants. En

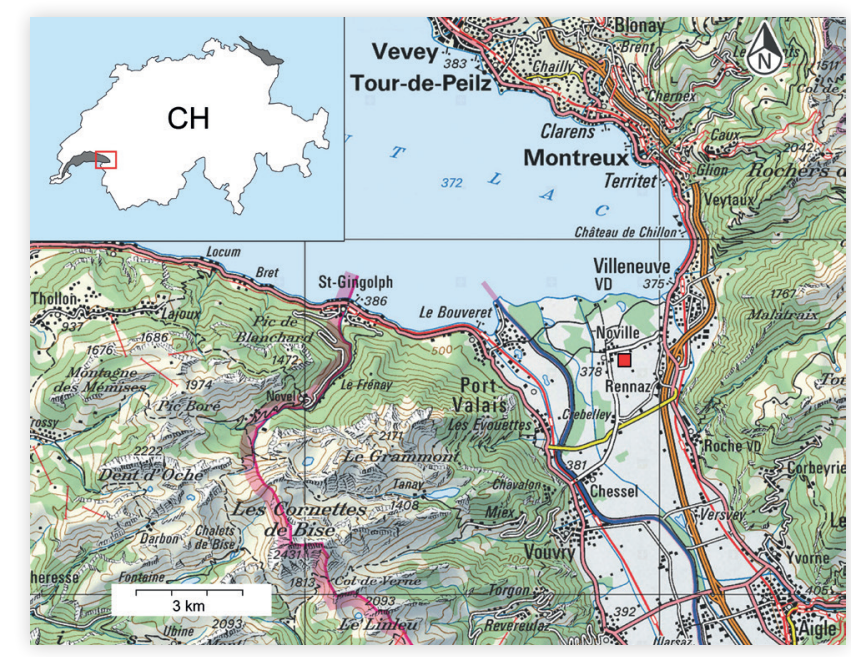

Figure 1 : situation géographique du site de RennazNoville, dans le sud-ouest de la Suisse. DAO M. Guélat.

résulte également un mouvement des eaux dans le lac sous forme d'une énorme vague qui balaye ses deux rives sur toute leur longueur et finit sa course au sein de la ville médiévale de Genève. Grégoire de Tours parle quant à lui plutôt d'un effondrement de la montagne qui anéantit le village de Tauredunum, installé vraisemblablement sur son flanc. La masse de terre et de gravats précipitée dans la plaine y bouche le cours du Rhône. Le fleuve inonde donc la vallée en amont avant de finalement surpasser la masse éboulée et de dévaler dans le lac, ce qui provoque le raz de marée. Les vestiges découverts

[1] Coordonnées : 46²2'55' $\mathrm{N} 6^{\circ} 54^{\prime} 17^{\prime \prime} \mathrm{E}$.

[2] RosselLi \& OLivier 2003, p. 407.

[3] Données du forage gazier Noville 1 réalisé en 2009, à environ $1 \mathrm{~km}$ au nord-ouest du site : communication orale de M. Weidmann, géologue.

[4] BADOUX 1965.

[5] Diamicton : terme général utilisé pour décrire un sédiment terrigène ou marin à classement médiocre ou nul, à constituants de granulométrie très variée et de provenance très large (Harland et al. 1966, p. 225256). Le mélange réalisé lors d'un glissement massif est une des origines possibles de ce sédiment hétérogène, semblable à un till (Van Vliet-Lanoë 2014, p. 361).

[6] SCHOENEICH et al. 2015, p. 162-165.

[7] KREMER et al. 2012, p. 756-757. 
sur le chantier du canal du Haut-Lac et leur contexte sédimentaire apportent ainsi les éléments de datation qui manquaient encore, du moins pour la partie terrestre de cet événement.

\section{MÉTHODES}

Un des objectifs de l'intervention de l'été 2018 consistait à préciser la stratigraphie des formations superficielles et leur géométrie. À l'exception des datations au radiocarbone [8], aucune analyse n'a été entreprise jusqu'à présent sur les sédiments prélevés. Cet article se concentre ainsi sur la chronostratigraphie et les découvertes archéologiques. Les processus de déformation observés sur le terrain ne sont que sommairement abordés.

Trois coupes ont fait l'objet d'un relevé à l'échelle $1 / 20$ au sein de l'emprise de fouille, ce qui représente $44 \mathrm{~m}$ linéaires au total (fig. 2). D'une hauteur moyenne avoisinant $2 \mathrm{~m}$, elles ont été localement approfondies par des forages à la tarière manuelle.

\section{RÉSULTATS}

\section{STRATIGRAPHIE}

Sous la surface de glissement, à -2,8 m de profondeur, se trouvent des silts très bien classés, carbonatés, saturés en eau et de consistance molle, qui passent à des sables laminés vers -3,5 m [9]. Ces silts non déformés, de teinte gris bleuté, renferment par endroits de la matière organique finement dispersée ou sous forme de débris végétaux, en particulier vers le sommet, sur 15 à $20 \mathrm{~cm}$ d'épaisseur, où la teneur en carbonates tend d'ailleurs à s'annuler. Ce dépôt s'est mis en place en milieu anoxique, probablement limnique, et l'accumulation de débris organiques au sommet signalent un net ralentissement de la sédimentation, voire un atterrissement.

[8] Datations AMS réalisées par le laboratoire ICA (International Chemical Analysis Inc.), Sunrise, États-Unis.

[9] Selon le forage F2 réalisé par l'entreprise Maric SA. Guichet cartographique de l'État de Vaud.

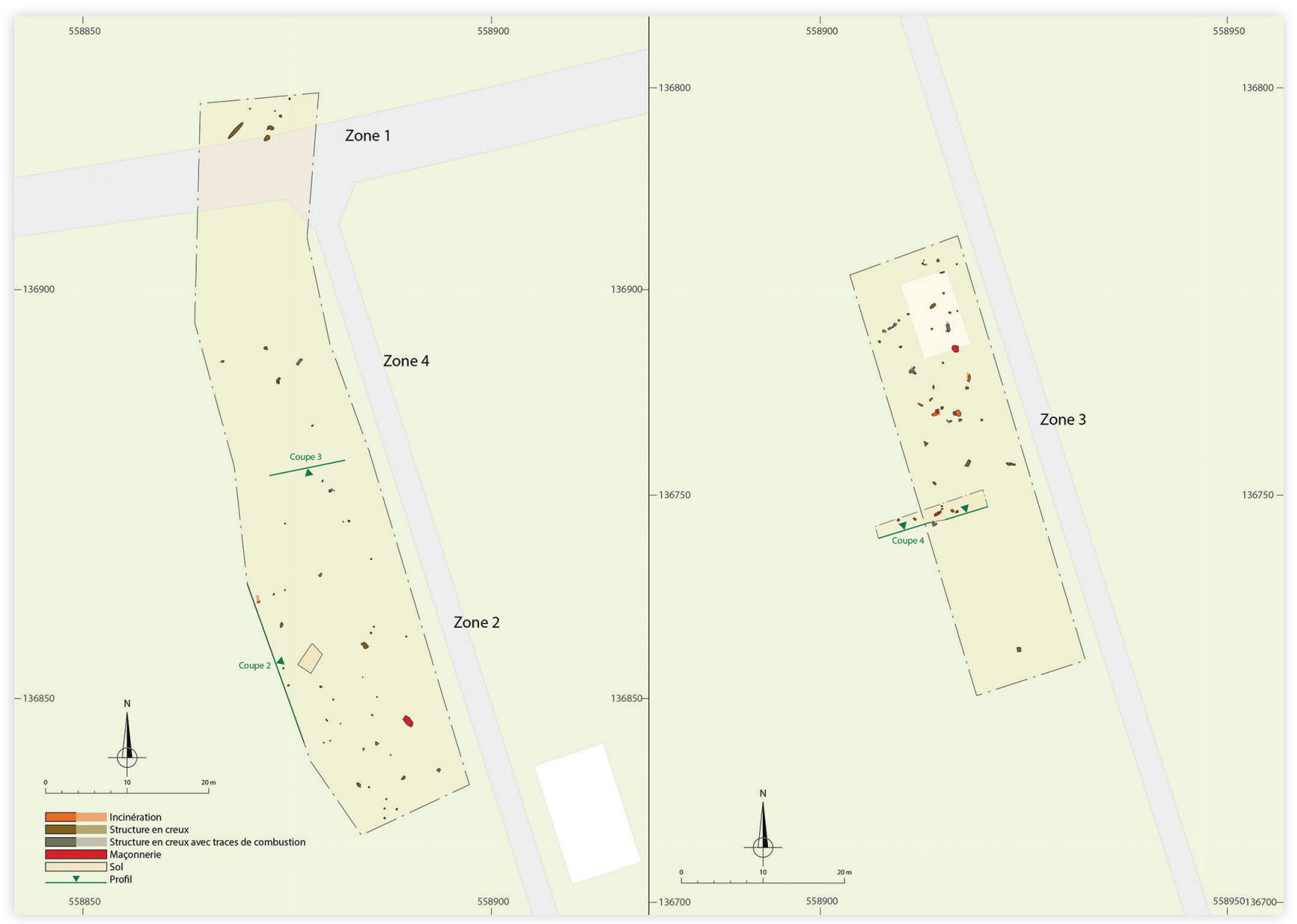

Figure 2 : plan de situation des vestiges et des coupes de référence dans les sections fouillées du canal du Haut-Lac. DAO A. Pignolet. 
Au-dessus du plan de glissement apparaissent souvent des graviers fluviatiles épais de $30 \mathrm{~cm}$ au maximum. Ils comprennent des éléments polygéniques, en général arrondis, et leur limite supérieure est déformée. Sur celle-ci vient se placer une formation hétérométrique d'une épaisseur assez constante, avoisinant les $2 \mathrm{~m}$. Elle se compose surtout de graviers épars enrobés d'une abondante matrice de sables silteux ou limoneux gris foncé, très ferme et fortement carbonatée. De nature diverse, les éléments sont en général arrondis, avec des blocs et cailloux subanguleux, dispersés dans la masse [10] (fig. 3). Au sein de celle-ci, des plissements d'amplitude métrique sont signalés par des lits de galets redressés, et des discontinuités verticales apparaissent localement. Cette unité contient aussi des artefacts isolés, voire des tronçons entiers de murs maçonnés (cf. infra 3.3.2). Il s'agit d'un diamicton formé principalement d'alluvions fines et grossières, mélangées au matériel d'un éboulement et, localement, à la démolition de vestiges archéologiques. En plus des graviers fluviatiles sous-jacents, d'autres unités sont incluses au matériel glissé sous forme d'enclaves déformées de sables et de silts gris bien triés, à litage distinct, parfois associées à des lambeaux de paléosols. Ces derniers se composent de limons humifères brun violacé, exempts de carbonates, renfermant souvent des fragments de terre cuite ou de céramique. Une succession de couches analogue à celle de ces enclaves a d'ailleurs été observée dans des compartiments considérés comme peu déformés.

La partie supérieure de la stratigraphie se constitue de limons plus sableux, voire gravillonneux à la base, de couleur brun-gris clair et d'une épaisseur allant de $10 \mathrm{~cm}$ à $1 \mathrm{~m}$. Leur contact inférieur avec le diamicton est érosif et rectiligne, et leur teneur en carbonates décroît vers le haut. Sur ces limons d'inondation, non concernés par la déformation, se place la terre végétale.

\section{LES COUPES DE RÉFÉRENCE}

Orientée sud-nord dans la zone 2, la coupe $n^{\circ} 2$ mesure $21 \mathrm{~m}$ de longueur et 2,5 $\mathrm{m}$ de hauteur (fig. 4). L'approfondissement de deux profils à chacune de ses extrémités a révélé la présence du plan de cisaillement sur les silts bleutés riches en matière organique. Au-dessus de ce contact anormal, dans la partie méridionale, apparaissent tout d'abord les graviers fluviatiles puis le diamicton comprenant des enclaves de sédiments laminés, très déformées, en convolutions et diapirs. À 2,3 $\mathrm{m}$ du bord sud de la coupe, une discontinuité verticale traverse le diamicton mais s'interrompt vers le haut, à la limite avec les limons d'inondation. Il doit s'agir d'une faille décrochante, délimitant les compartiments de la masse glissée. Dans la partie nord, une succession de silts et sables laminés, au sommet de laquelle s'est développé un paléosol tronqué renfermant des artefacts, semble peu déformée sur $7 \mathrm{~m}$ de long. Cette séquence est interprétée comme le remplissage d'un paléochenal, dont l'extension totale est inconnue.

D’axe ouest-est dans la zone 4, la coupe $n^{\circ} 3$ se trouve à une quinzaine de mètres au nord de la

[10] Un rapide examen pétrographique révèle que ceux-ci sont des calcaires siliceux, probablement du Lias, et des grès brun-gris, probablement du flysch.

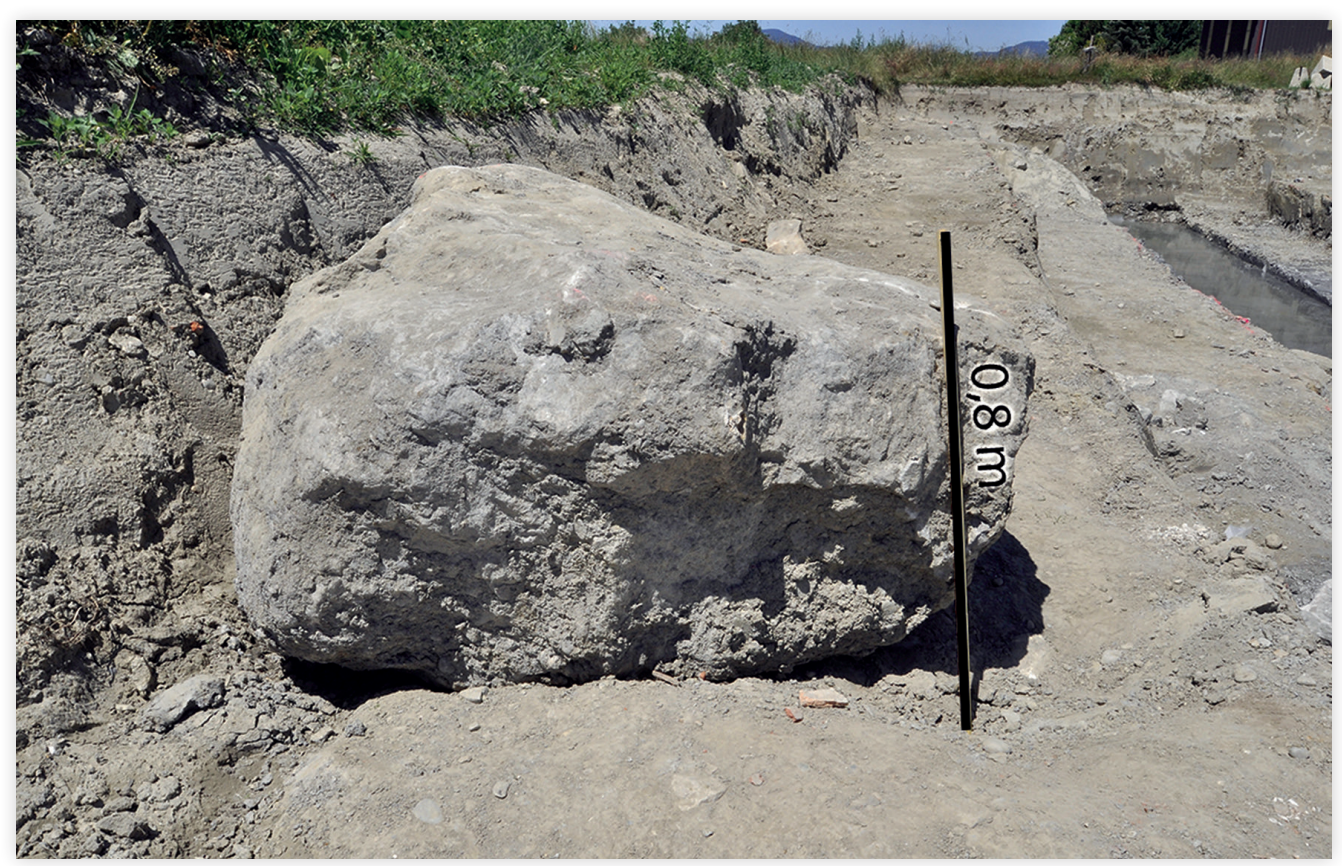

Figure 3

Bloc de calcaire siliceux dégagé à partir du matériel glissé. II s'agit d'un des plus grands éléments observés sur le site (hauteur $0,8 \mathrm{~m}$ ). Photo M. Millet. 

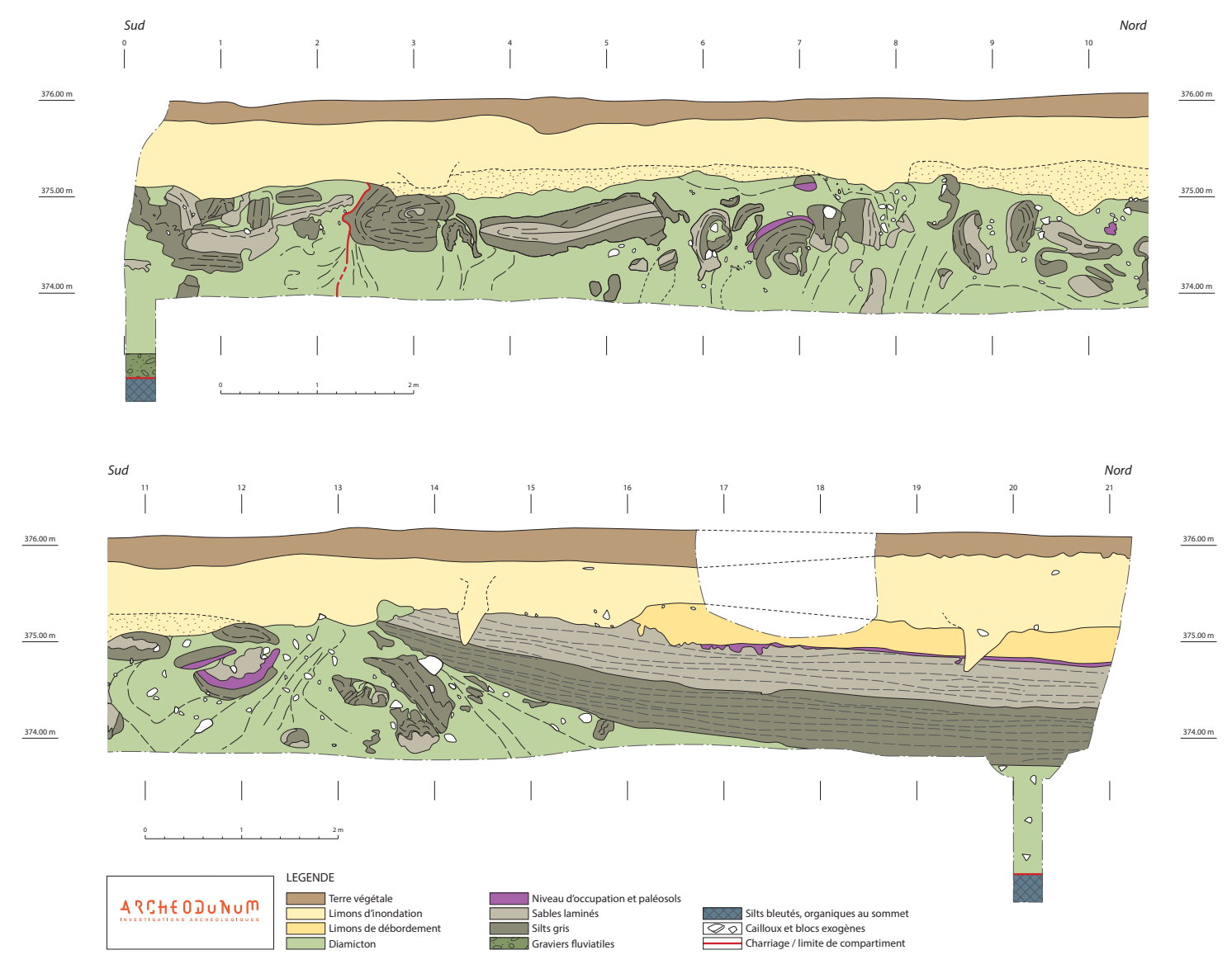

Figure 4 : relevé de la coupe $n^{\circ} 2$ en deux sections contiguës avec, dans la partie nord, le remplissage d'un paléochenal au sommet duquel s'est développé un paléosol, érodé. DAO A. Pignolet.

précédente. Sa longueur atteint $10 \mathrm{~m}$ et sa hauteur environ $2 \mathrm{~m}$, après un décapage superficiel d'environ $1 \mathrm{~m}$ d'épaisseur, soit jusqu'au sommet du diamicton (fig. 5). Ce dernier renferme des fragments de tuile épars et on y trouve, comme au sein de la coupe $n^{\circ} 2$, les enclaves de silts et surtout de sables bien triés. Parmi les figures de déformation apparentes, on identifie des plis couchés souvent rompus, déversés vers l'ouest. Dans la partie orientale de la coupe, une de ces charnières inclut un lambeau de paléosol, alors que sa base s'appuie sur les graviers fluviatiles, déformés en conformité. Plus bas, le plan de cisaillement accuse un léger pendage vers I'ouest. Il met en contact les graviers rhodaniens avec les silts bleutés, qui ne recèlent ici qu'une matière organique diffuse.

La coupe $n^{\circ} 4$ enfin se déploie d'est en ouest, dans la zone 3, à plus de $120 \mathrm{~m}$ au sud des deux précédentes (fig. 5). Recoupée par un sondage exploratoire dans sa partie médiane, elle a une longueur totale de $13 \mathrm{~m}$ et une hauteur maximale de 1,6 m. En profondeur, la surface de glissement n'a pas été atteinte et le diamicton apparaît sans équivoque sous les limons d'inondation. Il contient à nouveau des enclaves de silts laminés grisâtres, incluant localement des lambeaux de paléosol(s). Les figures de déformation apparentes montrent bien des plis déversés, mais vers l'est cette fois. Plus abondant que dans les autres coupes, du mobilier archéologique tel que des fragments de tuile et de céramique, avec des petits blocs calcaires probablement équarris, s'y distingue en plus des habituels galets fluviatiles et cailloux anguleux.

\section{VESTIGES ARCHÉOLOGIQUES ET MOBILIER}

L'intervention préventive réalisée a permis de reconnaître une centaine de structures potentielles (fig. 2), d'abord prudemment qualifiées d'anomalies eu égard au contexte sédimentaire perturbé. Il s'agit essentiellement de vestiges d'occupations en matériau périssable (trou de poteau), parfois plus pérennes (maçonneries). La présence d'un espace funéraire est aussi pressentie. Le croisement des données stratigraphiques, des datations au radiocarbone et des études de mobilier a mis en évidence deux périodes de fréquentation, I'une protohistorique et I'autre gallo-romaine. Au vu du contexte largement perturbé et des relations lacunaires entre les structures, l'élaboration d'un phasage plus précis n'a pas été possible. 


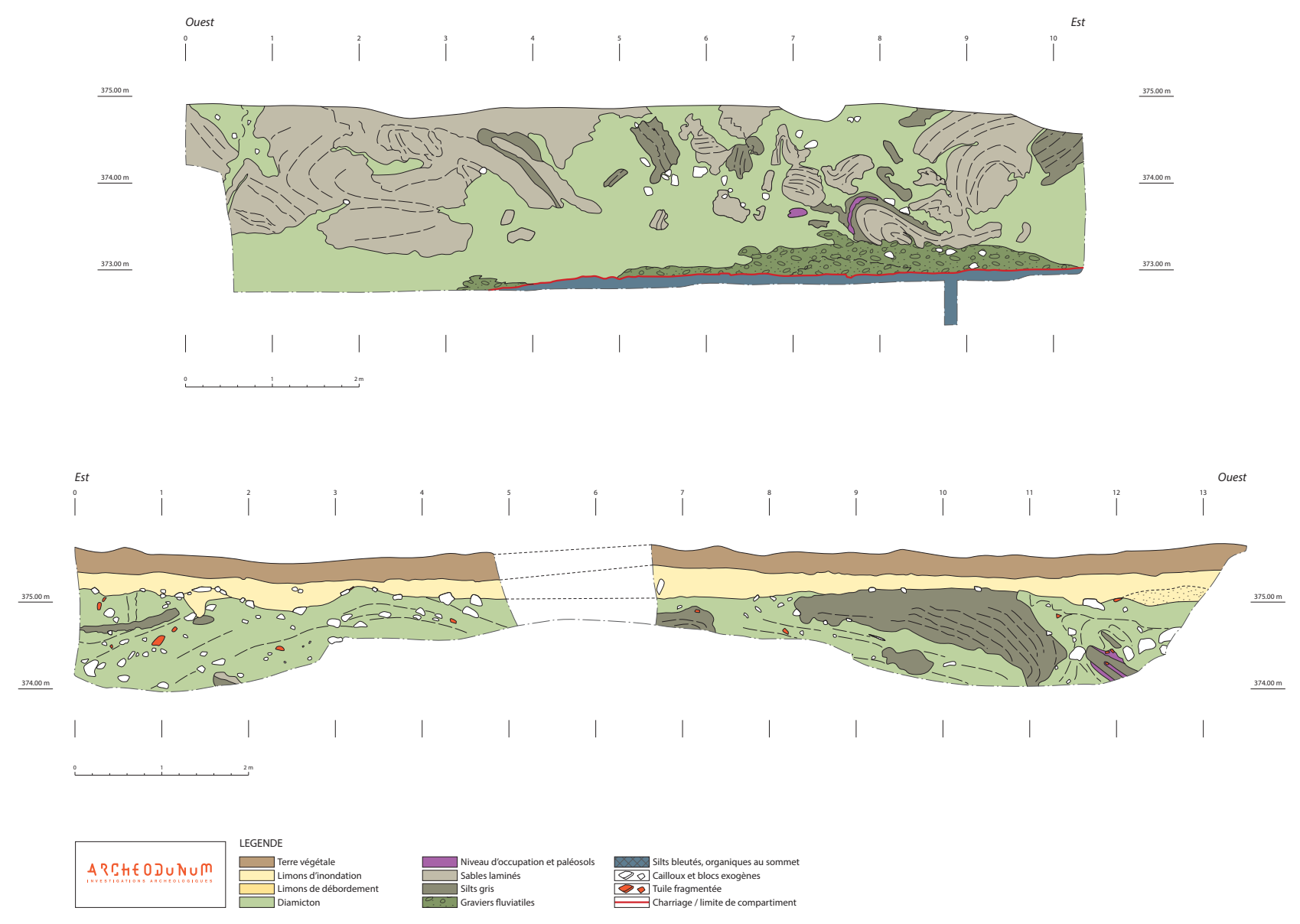

Figure 5 : relevés des coupes $n^{\circ} 3$ (en haut) et 4 (en bas). La première met au jour le plan de glissement à la base et montre, au-dessus, les déformations du diamicton. La seconde révèle que la masse glissée renferme de nombreux artefacts tels que des fragments de tuile ou de céramique, et des petits blocs calcaires probablement équarris. DAO A. Pignolet.

\section{L'occupation protohistorique}

La fréquentation du site à la protohistoire est attestée par l'unique présence d'un niveau de circulation composé de graviers de calcaire jaune très indurés. D'une épaisseur moyenne de 5 $\mathrm{cm}$, il a été observé notamment à la faveur d'un compartiment sédimentaire non perturbé présent au nord-ouest de la zone 2 et relevé sur la coupe de référence $n^{\circ} 2$. Sa position stratigraphique permet d'assurer son antériorité aux niveaux galloromains, qui le recoupent ponctuellement. De plus, la fouille de son tronçon préservé a permis la collecte de plusieurs fragments de céramiques attribuables à la période de La Tène (céramique à vernis noir). Ce niveau d'occupation se retrouve aussi de manière éparse sur toute l'emprise du chantier, à l'état de lambeaux dans le diamicton. Une datation radiocarbone sur un charbon prélevé dans le cailloutis du compartiment conservé donne un intervalle de 200-40 cal BC (ICA18C/1057).

On peut aussi noter la découverte, au sein de la masse transportée, de quelques tessons de céramique protohistorique, d'amphore Dressel 1 , de trois monnaies d'émission républicaine (un as, un quinaire et un denier de Marcus Vargunteius) et d'un bouton mouluré de passe-guide de type Hoppstädten, daté généralement de La Tène finale. Ces éléments laissent donc présager d'une occupation laténienne du secteur, oblitérée par les implantations et les mouvements de terrains postérieurs.

Cette présence était déjà supposée sur la base de la découverte, à environ $1 \mathrm{~km}$ au nord du chantier, d'une effigie en chêne datée de la fin de La Tène sur la base de trois monnaies déposées dans le tronc: un quinaire gaulois et deux oboles [11].

\section{L'occupation du Haut-Empire}

La grande majorité des vestiges archéologiques rencontrés concerne la période gallo-romaine. Le matériel collecté (fig. 10) et les analyses ${ }^{14} \mathrm{C}$ réalisées abondent dans le sens d'une occupation comprise semble-t-il entre le milieu du Ier et le courant du IVe siècle de notre ère.

[11] PAUNIER 1998, p. 99. 

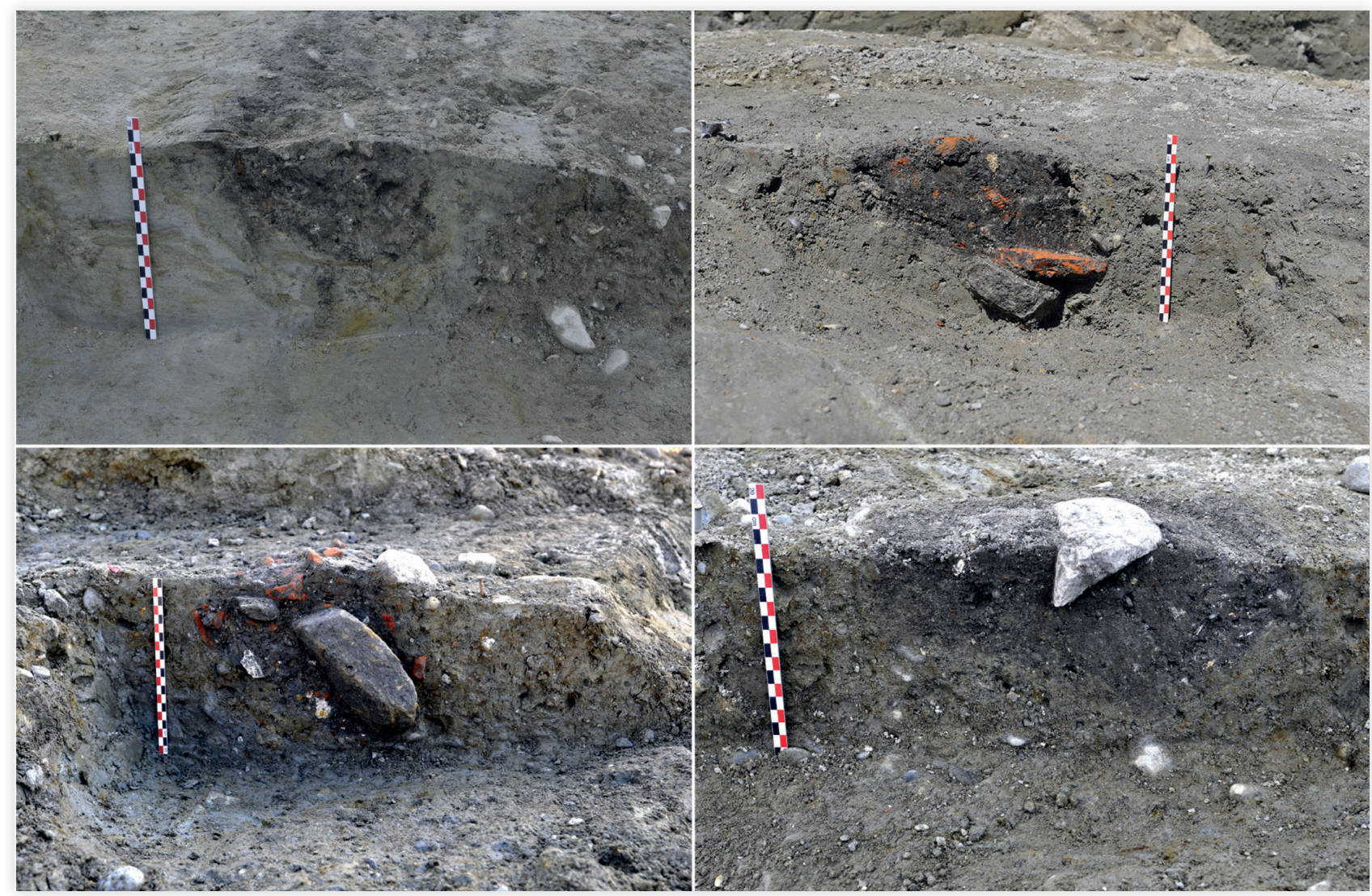

Figure 6 : coupes de trous de poteaux non déformés. Photos M. Millet.

Il s'agit principalement de structures fossoyées telles que I'on peut en trouver sur les sites livrant des empreintes d'architecture en matériau périssable. Il est cependant difficile de les distinguer avec certitude. En effet, l'ensemble des vestiges étant implanté dans un contexte sédimentaire largement perturbé, cela se traduit par des morphologies et profils de structures majoritairement irréguliers. Néanmoins, plusieurs trous de poteaux ont été reconnus à la faveur de profils de creusement réguliers et de la présence de blocs de calages (fig. 6), mais aucun plan de bâtiment n'est avéré.
À l'inverse, on a pu observer de nombreuses anomalies, que l'on a d'abord interprétées comme des structures en creux, mais qui se sont révélées être des lambeaux de sols pris dans la déformation (fig. 7). Cette discrimination entre structures fossoyées et lambeaux de sols déformés s'est également faite grâce à l'observation fine de la séquence encaissante de chaque anomalie documentée, sur la base de l'analyse des coupes de références réalisée en amont.

En parallèle des empreintes d'installations périssables, plusieurs témoins de constructions maçonnées ont été repérés. Il s'agit d'élévations

\section{Figure 7}

Coupe de lambeaux de sols déformés. Photo M. Millet.

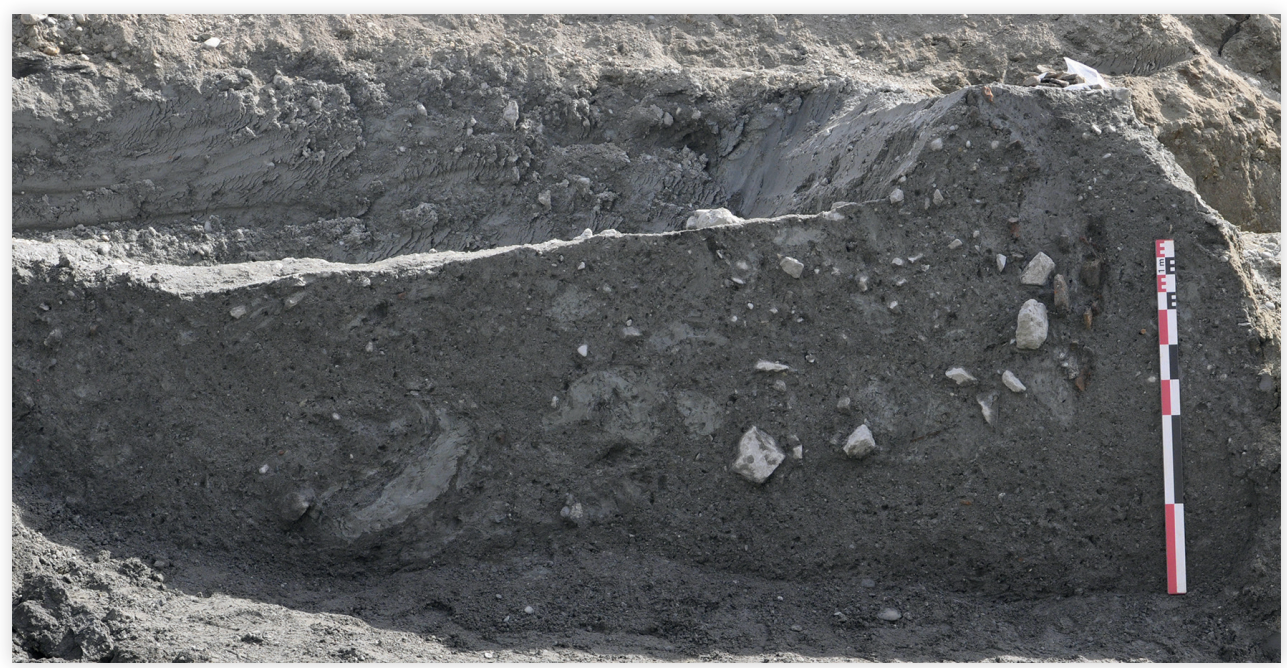




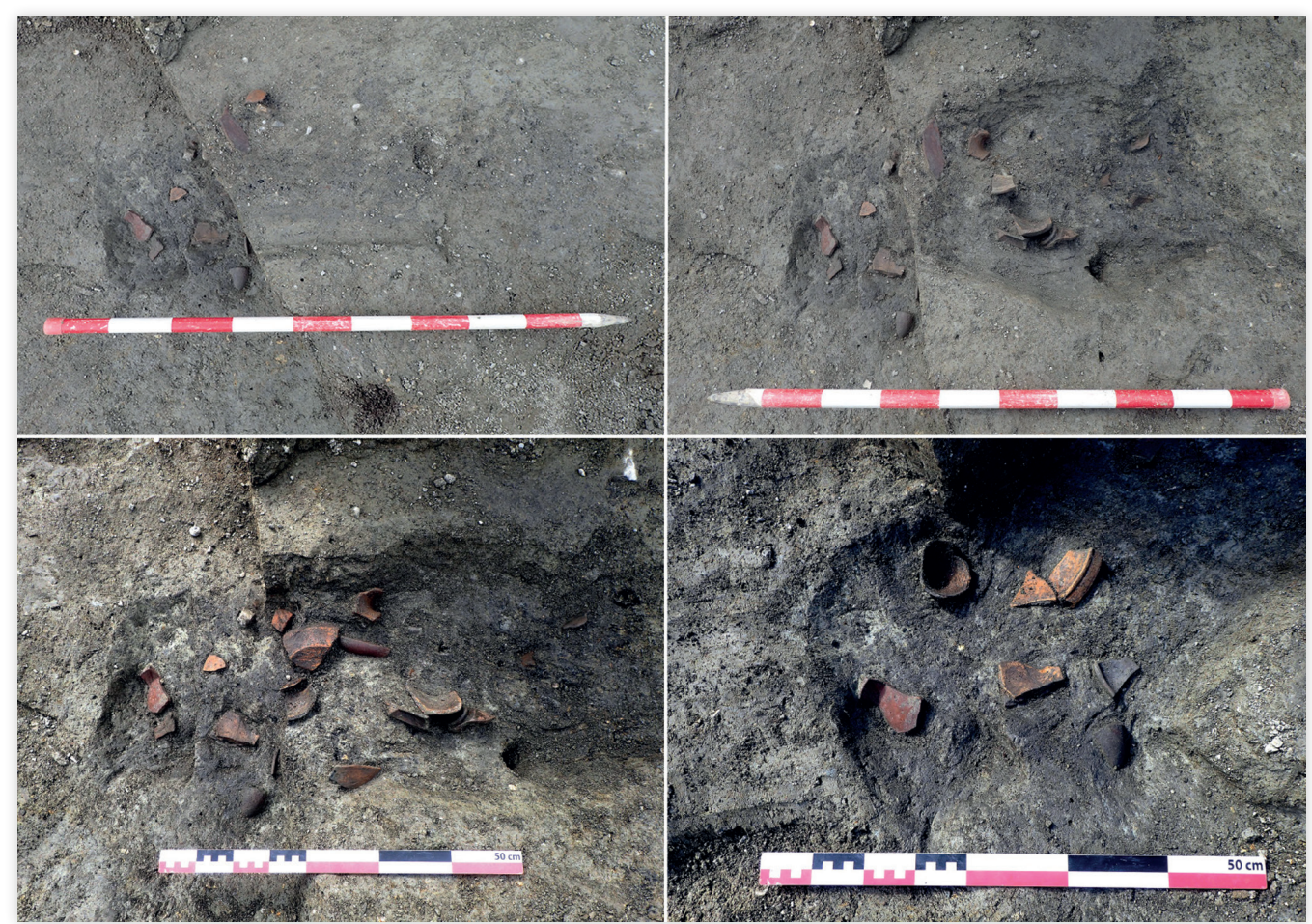

Figure 8

Vues en cours de fouille de l'incinération ST1. Photos M. Millet. partiellement préservées en pans et souvent disposées obliquement dans la matrice perturbée. Au nombre de sept, ces reliquats d'élévations ne présentent pas les mêmes caractéristiques et états de conservation. Trois d'entre eux se résument à des concentrations lâches de blocs, dont certains peuvent être liés au mortier de chaux. Malgré la présence de pierres de taille jointes dans un contexte de conservation pourtant défavorable, leur interprétation en tant que restes de démolition apparaît plus probable.

Les quatre autres représentants du gros-œuvre sont moins altérés et présentent une élévation de plusieurs assises (fig. 9). La maçonnerie la plus importante du site présente une longueur de $1,5 \mathrm{~m}$ pour une largeur de $0,65 \mathrm{~m}$ avec le ressaut de fondation. Elle se compose de blocs et boulets grossièrement équarris. Sa fondation comprend trois assises conservées sur une hauteur de 0,36 m (calibre des blocs de 0,1 à 0,4 m), liées d'un mortier blanc jaunâtre très sableux et friable. L'élévation à proprement parler est conservée sur trois assises également. Ce mur est fait d'un appareil courant (panneresse) composé de blocs calibrés $(0,15$ à 0,2 m) agrégés à l'aide d'un mortier blanc plus compact que pour la fondation. D'orientation sud-est/nordouest, il se trouve déversé vers l'ouest. Au niveau du ressaut de fondation sur la largeur sud, on peut observer une plaque de mortier lissé installée sur une assise de réglage en tuiles. Elle se situe dans le prolongement de la première assise et pourrait
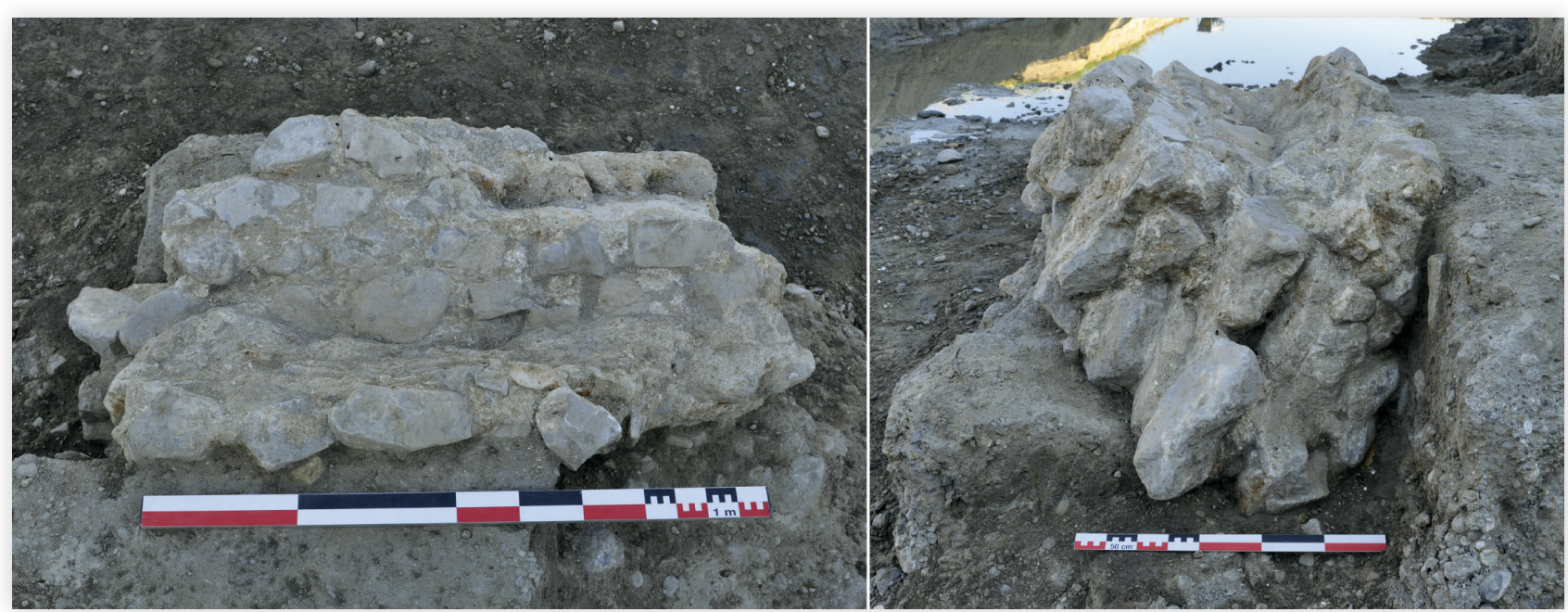

Figure 9 : maçonnerie du site de Noville. Photos M. Millet. 
correspondre au reliquat d'un seuil ou d'un sol maçonné.

Les trois éléments de construction restant ont des mensurations comprises entre 0,8 et $0,5 \mathrm{~m}$ de longueur et entre 0,8 et 0,4 m de largeur, pour une élévation de deux à quatre assises.

Ces murs ne se trouvent donc pas à proprement parler in situ, mais leur état de conservation semble indiquer une provenance proche, avec selon toute vraisemblance la présence d'un établissement gallo-romain à proximité directe de la zone investiguée. Enfin, plusieurs structures funéraires ont également été reconnues. Il s'agit de seize fosses pouvant être liées au rite de la crémation. La plupart ne sont que partiellement conservées, à l'exception d'une, préservée à la faveur d'une enclave sédimentaire non déformée (fig. 8). Ces structures possèdent un comblement charbonneux et contiennent du mobilier calciné (céramique, verre, clous en fer) et/ou des esquilles osseuses animales et humaines. Mais seulement quatre d'entre elles présentent une association entre mobilier calciné et restes humains. Leur état de conservation fragmentaire ne permet pas d'en apprendre plus sur leur nature exacte [12].

\section{ÉLÉMENTS DE DATATION}

Une série de cinq datations ${ }^{14} \mathrm{C}$ a été effectuée sur des matières organiques prélevées en dessous du cisaillement, dans les silts bleutés (fig. 11). L'intervalle qu'elles définissent, plutôt cohérent avec la position altimétrique des échantillons, va de 2880 cal BC (ICA19W/0403), âge obtenu sur un tronc d'arbre découvert au sud de l'emprise de fouille, à 430 cal AD (ICA19W/0969), date provenant d'un fragment de bois prélevé dans la zone 4. De plus, la pointe d'un pieu implanté dans ces mêmes dépôts limniques détermine la limite chronologique (TAQ) pour leur mise en place à 250-400 cal AD (ICA19W/0338). Cette borne supérieure est d'ailleurs confirmée par la date de 250-430 cal AD obtenue sur un macroreste (ICA18C/1058).

Au sein du compartiment peu déformé de la partie nord de la coupe $n^{\circ} 2$, le paléosol du haut de la séquence a livré par analyse ${ }^{14} \mathrm{C}$ un âge de $200-40$ cal BC (ICA18C/1057), en accord avec la typologie du mobilier. En particulier, le monnayage républicain et la céramique à vernis noir garantissent l'insertion de ce niveau anthropisé dans le Second âge du Fer (période de La Tène finale), qui correspond ainsi à la limite chronologique inférieure (TPQ) pour la mise en place de la masse glissée.

De plus, quatre dates proviennent de structures archéologiques déformées de la zone 3, décapées au sommet du diamicton (ICA18C/1052, /1054, /1055 et /1056). Elles définissent une fourchette chronologique d'environ 100-400 cal AD, confortée par la typologie du mobilier qui place cette occupation gallo-romaine entre la deuxième partie du $\mathrm{I}^{\mathrm{er}}$ et la fin du IVe siècle ap. J.-C. Ces éléments rajeunissent donc la limite chronologique inférieure de l'événement catastrophique de près d'un demi-millénaire. Enfin, du mobilier moderne (tessons de céramique vernissée, casseroles et serrures) a été découvert dans les limons d'inondation scellant le diamicton.

[12] Pour l'analyse détaillée, voir le rapport d'intervention : MiLLET 2020

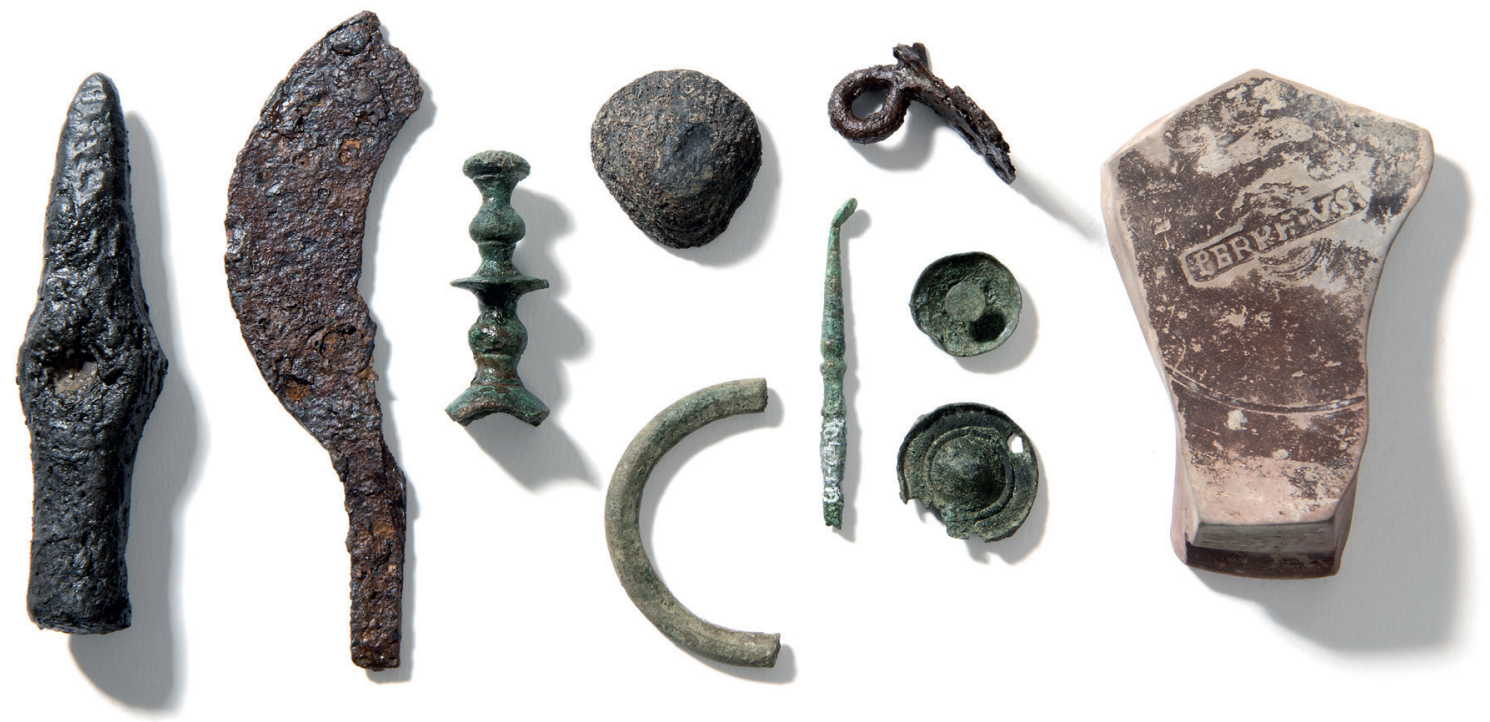

Figure 10 : sélection d'artefacts découverts lors de la fouille. Photo N. Jacquet. 


\begin{tabular}{|c|c|c|c|c|}
\hline $\begin{array}{l}\text { Réf. } \\
\text { laboratoire }\end{array}$ & Matière & Coordonnées & $\begin{array}{l}\text { Age } 14 \mathrm{C} \mathrm{BP} \\
\text { conv. }\end{array}$ & $\begin{array}{l}\text { Date calibrée } \\
2 \text { sigma }\end{array}$ \\
\hline ICA18C/1052 & Charbon de bois & $\begin{array}{l}\text { Zone } 1, \text { ST17, } \\
\text { trou de poteau, } \\
\text { alt. } 374.26 \mathrm{~m}\end{array}$ & $1830+/-30 \mathrm{BP}$ & $\begin{array}{l}80-260 \text { cal AD ( } 95.0 \%) \\
300-320 \text { cal AD }(0.4 \%)\end{array}$ \\
\hline ICA $18 C / 1054$ & Charbon de bois & $\begin{array}{l}\text { Zone 2, ST44, } \\
\text { trou de poteau, } \\
\text { alt. } 374.87 \mathrm{~m}\end{array}$ & $1810+/-30 \mathrm{BP}$ & $\begin{array}{l}120-260 \text { cal AD ( } 88.6 \%) \\
280-330 \text { cal AD }(8.8 \%)\end{array}$ \\
\hline ICA18C/1055 & Charbon de bois & $\begin{array}{l}\text { Zone } 4 \text {, ST91, } \\
\text { trou de poteau, } \\
\text { alt. } 374.48 \mathrm{~m}\end{array}$ & $1770+/-30 \mathrm{BP}$ & $130-350 \mathrm{cal} A D$ \\
\hline ICA18C/1056 & Charbon de bois & $\begin{array}{l}\text { Zone 4, ST94, } \\
\text { trou de poteau, } \\
\text { alt. } 374.23 \mathrm{~m}\end{array}$ & $1750+/-30 \mathrm{BP}$ & $220-390 \mathrm{cal} A D$ \\
\hline ICA18C/1057 & Charbon de bois & $\begin{array}{l}\text { Coupe } 2 \text { partie nord, } \\
\text { paléosol, } \\
\text { alt. } 374.80 \mathrm{~m}\end{array}$ & $2090+/-30$ BP & 200- 40 cal BC \\
\hline ICA $18 C / 1058$ & Reste végétal & $\begin{array}{l}\text { Coupe } 2 \text {, silts } \\
\text { organiques, } \\
\text { alt. } 372.70 \mathrm{~m}\end{array}$ & $1670+/-30 \mathrm{BP}$ & $\begin{array}{l}250-300 \text { cal AD }(6.4 \%) \\
320-430 \text { cal AD }(89.0 \%)\end{array}$ \\
\hline ICA19W/0338 & Bois & $\begin{array}{l}\text { Zone } 2 \text {, pointe } \\
\text { de pieu dans silts } \\
\text { bleutés, alt. } 372.82 \mathrm{~m}\end{array}$ & $1710+/-30 \mathrm{BP}$ & $250-400 \mathrm{cal} \mathrm{AD}$ \\
\hline ICA19W/0403 & Bois & $\begin{array}{l}\text { Tronc dans silts } \\
\text { bleutés }\end{array}$ & $4140+/-40$ BP & $2880-2580 \mathrm{cal} \mathrm{BC}$ \\
\hline ICA190S/0967 & Sédiment & $\begin{array}{l}\text { Coupe } 2 \text { partie sud, } \\
\text { silts organiques, } \\
\text { alt. } 372.90 \mathrm{~m}\end{array}$ & $2020+/-30 \mathrm{BP}$ & $110 \mathrm{BC}-80 \mathrm{cal} A D$ \\
\hline ICA19C/0968 & Charbon de bois & $\begin{array}{l}\text { Coupe } 3 \text { partie est, } \\
\text { silts organiques, } \\
\text { alt. } 372.95 \mathrm{~m}\end{array}$ & $2030+/-30 B P$ & $180 \mathrm{BC}-70 \mathrm{cal} A D$ \\
\hline ICA19W/0969 & Bois & $\begin{array}{l}\text { Zone } 4 \text {, silts bleutés, } \\
\text { près de coupe } 3 \text {, } \\
\text { alt. } 372.65 \mathrm{~m}\end{array}$ & $1680+/-30 \mathrm{BP}$ & $\begin{array}{l}250-300 \text { cal AD }(10,2 \%) \\
320-430 \text { cal AD }(85,2 \%)\end{array}$ \\
\hline
\end{tabular}

Figure 11

Datations ${ }^{14} \mathrm{C}$ réalisées pour le site de Rennaz-Noville à partir d'échantillons dont les caractéristiques sont précisées.

\section{DISCUSSION}

\section{INTERPRÉTATION DES FIGURES SÉDIMENTAIRES}

Selon les figures de déformation observées dans le diamicton, la contrainte à l'origine du cisaillement se serait exercée principalement d'ouest en est, en accord avec les données de la littérature [13]. En effet, la coupe $n^{\circ} 2$ d'axe sud-nord recoupe les charnières des plis alors que la coupe $n^{\circ} 4$, orientée est-ouest, révèle que ces derniers sont en général couchés vers l'est. Dans la coupe $n^{\circ} 3$ d'axe ouestest, il semble que la masse déplacée ait subi une déformation secondaire comme le montre la limite replissée entre les graviers fluviatiles et le diamicton. Ce « rétrocharriage » expliquerait l'apparent déversement des plis vers I'ouest dans la zone 4, au nord du périmètre exploré. Quant au découpage en compartiments plus ou moins déformés, il pourrait

[13] SCHOENEICH et al. 2015, p. 163. 
indiquer que le transport s'est effectué grâce à un fond fluidifié lors de l'écroulement. Les sédiments alluviaux ou limniques détrempés sont en effet susceptibles de se liquéfier sous l'effet de la surpression et peuvent ainsi jouer le rôle de lubrifiant sous la masse écroulée [14].

\section{DISPERSION DES VESTIGES ARCHÉOLOGIQUES}

Suite à la perte de portance du sous-sol, des édifices résistants peuvent s'enfoncer et se renverser. La découverte sur le site de plusieurs tronçons de maçonneries basculés d'époque gallo-romaine est rapportée à ce type de phénomène, au cours duquel un ou plusieurs bâtiments, peut-être déjà en ruine, ont été complètement démolis. Ces sections de murs, avec leurs fondations encore solidaires parfois, ont été mises au jour jusqu'à une profondeur proche de $2 \mathrm{~m}$ sous la surface actuelle, au sein du diamicton. On ne s'étonnera donc pas de la grande dispersion spatiale du mobilier archéologique. Il en va de même pour les structures fossoyées (trous de poteau, incinérations), le paléosol protohistorique et le niveau d'occupation du HautEmpire inclus sous forme de lambeaux à la masse transportée, dans la zone 2 tout spécialement.

\section{SIGNIFICATION DES ÉLÉMENTS DE DATATION}

Les dates obtenues à partir d'échantillons de sédiment et de charbon de bois situés juste sous le plan de glissement indiquent que les silts organiques se sont accumulés jusqu'au début de notre ère au plus tard. Mais les fragments végétaux qu'ils contiennent sont en général plus récents. En particulier, I'âge d'un pieu probablement cisaillé sous la masse de débris concorde avec celui d'un macroreste, matière considérée comme très fiable pour les datations. Ces deux dates définissent ainsi un intervalle allant du milieu du III $^{\mathrm{e}}$ au début du Ve siècle. Au-dessus des silts organiques se trouvent soit des graviers fluviatiles non datés, soit le diamicton. Ce dernier inclut des sédiments plus anciens que le déplacement, sous forme d'enclaves perturbées ou, au contraire, d'îlots apparemment peu déformés, à l'instar de la séquence au sommet de laquelle se trouve un niveau de la Tène finale, en coupe $n^{\circ} 2$. La borne supérieure de l'intervalle que définissent les datations des structures gallo-romaines et la typologie du mobilier associé $s^{\prime}$ insère vers la fin du IVe siècle après J.-C. Il s'agit là d'une limite chronologique inférieure pour la mise en place du diamicton. Les dépôts de plaine alluviale recouvrant la masse glissée n'ont pas été datés. Le rare mobilier qu'on y a découvert tend à indiquer que ces limons se sont déposés dès le Moyen Âge au plus tôt, et surtout durant la période moderne.

\section{INSERTION CHRONOLOGIQUE DE LA CATASTROPHE}

Les résultats des datations ${ }^{14} \mathrm{C}$ et de la chronotypologie sont donc concordants et permettent d'affirmer que l'avalanche rocheuse reconnue à Noville a eu lieu après un intervalle compris entre le milieu du III $^{\mathrm{e}}$ et le début du Ve siècle. Selon les sources écrites, celle du Tauredunum a eu lieu au moins un à trois siècles plus tard. Pourtant, plusieurs arguments parlent en faveur d'un seul et même événement. En effet, il est statistiquement très peu probable que deux catastrophes naturelles d'une telle ampleur, soit d'un volume estimé entre 20 et 30 millions de mètres cubes [15], aient pu avoir lieu au même endroit, dans un laps de temps séculaire. De plus, si un écroulement précède de peu celui de l'an 563, son matériel aurait été oblitéré par ce dernier, reconnu comme majeur, et ne serait de fait plus observable. Dans le même ordre d'idées, la conservation d'un compartiment non perturbé, comprenant un niveau de l'âge du Fer, paraît impossible après deux écroulements aussi importants. Enfin et surtout, l'analyse de carottes forées dans le fond du lac Léman montrent qu'il n'y a pas eu de transport en masse dans ce milieu durant les deux à trois siècles ayant précédé la catastrophe du Tauredunum [16].

\section{CONCLUSIONS}

L'étude menée sur le chantier du Canal du HautLac fournit de nouveaux acquis concernant la « formation de Chessel-Noville » et I'occupation humaine dans la vallée du Rhône, à proximité de son ouverture sur le lac Léman. La chronologie de la mise en place de ce dépôt d'écroulement est maintenant précisée grâce à des éléments de datation provenant de datations ${ }^{14} \mathrm{C}$ et de la chronotypologie, qui montrent une remarquable cohérence. Deux occupations humaines distinctes ont été identifiées sur

[14] Parriaux 2006, p. 92 ; PedrazZini et al. 2013, p. 65.

[15] SCHOENEICH et al. 2015, p. 171.

[16] KREMER et al. 2015, p. 1323. 
le site. La première, plutôt discrète, se rapporte au Second âge du Fer et la suivante, nettement plus étendue, à la période gallo-romaine. La déformation des niveaux et structures archéologiques, de même que l'enfoncement spectaculaire de maçonneries parfois basculées, résultent d'une liquéfaction de leur soubassement. La contrainte s'est exercée d'ouest en est, comme le montrent les structures sédimentaires de la masse glissée qui inclut des blocs détachés du versant en rive gauche du Rhône. Ceux-ci ont donc traversé la plaine alluviale, sur une distance de 3,5 km. En définitive, l'ensemble de ces résultats géoarchéologiques sont compatibles avec une mise en place du matériel glissé lors de la catastrophe du Tauredunum, qui a eu lieu, nous I'avons vu, vers le milieu du VIe siècle.

\section{REMERCIEMENTS}

De sincères remerciements s'adressent à Sébastien Freudiger (Archeodunum SA) et Nicole Pousaz (archéologue cantonale de l'État de Vaud) pour avoir facilité les travaux entrepris. Merci aussi à Robert Fellner pour la révision du résumé en anglais. Merci enfin aux relecteurs anonymes pour leurs suggestions.

\section{BIBLIOGRAPHIE}

BadouX, Héli, 1965, Feuille 47 Montreux (CN1264). Atlas géologique de la Suisse 1 :25’000, Bern.

harland, Walter Brian, Herod, Kay N. \& Krinsley, David Henry, 1966, « The definition and identification of tills and tillites », Earth-Science Reviews 2, p. 225-256.

Kremer, Katrina, Simpson, Guy \& Girardclos, Stéphanie, 2012, « Giant Lake Geneva Tsunami in AD 563 », Nature Geoscience 5/11, p. 756-757.

Kremer, Katrina, Hilbe, Michael, Simpson, Guy, Decrouy, Laurent, Wildi, Walter \& Girardclos, Stéphanie, 2015, « Reconstructing 4000 years of mass movement and tsunami history in a deep peri-Alpine lake (Lake Geneva,France-Switzerland) », Sedimentology 62, p. 1305-1327.

Parriaux, Aurèle, 2006, Géologie. Bases pour l'ingénieur, Lausanne.

MILlet, Morgan, 2020, "Noville - Canal du Haut-Lac", Rapport final d'opération archéologique, Gollion.

Paunier, Daniel, 1998, « Le Léman, de l'époque gallo-romaine au Moyen Âge », Archives des sciences et compte rendu des séances de la Société 51, p. 91-102.

Pedrazzini, Andrea, Jaboyedoff, Michel, Loye, Alexandre \& DerRon, Marc-Henry, 2013, « From deep seated slope deformation to rock avalanche: Destabilization and transportation models of the Sierre landslide (Switzerland) », Tectonophysics 605, p. 149-168.

Rosselli, Alberto \& Olivier, Raymond, 2003, « Modélisation gravimétrique 2.5D et cartes des isohypses au $1: 100$ '000 du substratum rocheux de la Vallée du Rhône entre Villeneuve et Brigue (Suisse) », Ecologae Geologicae Helvetiae 96/3, p. 399-423.

Schoeneich, Philippe, Weidmann, Marc \& Blomjous, Carole, 2015, « L'énigme du Tauredunum enfin résolue? », dans Emmanuel Reynard et al. (éd.), Le Rhône, entre nature et société, Sion, p. 153-174.

VAN Vliet-Lanö̈, Brigitte, 2014, Les environnements froids. Glaciaire et périglaciaire, Paris. 